\title{
STEAP4 regulates focal adhesion kinase activation and CpG motifs within STEAP4 promoter region are frequently methylated in DU145, human androgen-independent prostate cancer cells
}

\author{
TAKAHIKO TAMURA ${ }^{1,2}$ and JOE CHIBA ${ }^{1}$ \\ ${ }^{1}$ Department of Biological Science and Technology, Tokyo University of Science, \\ 2641 Yamazaki, Noda-shi, Chiba 278-8510, Japan
}

Received June 19, 2009; Accepted July 22, 2009

DOI: 10.3892/ijmm_00000270

\begin{abstract}
The possible roles of STEAP4 in cancer progression have not been reported. In this study, we report that STEAP4 expression is able to inhibit anchorage-independent cell growth. We also demonstrate that STEAP4 associates with focal adhesion kinase (FAK) and regulate the activity of FAK through Y397 phosphorylation. Furthermore, we show that $\mathrm{CpG}$ sequences in STEAP4 promoter region were frequently methylated in DU145, androgen-independent prostate cancer cells. Demethylation treatment induced STEAP4 expression in DU145, suggesting the possibility that STEAP4 expression in cancer cells is in part epigenetically regulated. Collectively, these data demonstrate a novel function of STEAP4 and that STEAP4 may play an important role in tumor malignancy.
\end{abstract}

\section{Introduction}

STEAP4 (six transmembrane epithelial antigen of the prostate 4) is a member of the STEAP family (1). Structurally, STEAP4 (also called STAMP2) is a 459 amino acid protein characterized by a molecular topology of six transmembrane domains. The cytoplasmic N-terminal domain of STEAP4 shows structural similarity with bacterial and archaeal FNO oxidoreductase and STEAP4 exhibits a strong iron reductase activity (2). The analysis of STEAP4

Correspondence to: Dr Takahiko Tamura, Department of Biological Science and Technology, Tokyo University of Science, 2641 Yamazaki, Noda-shi, Chiba 278-8510, Japan

E-mail: tktamura@rs.noda.tus.ac.jp

Present address: ${ }^{2}$ Department of Molecular Microbiology and Immunology, Graduate School of Biomedical Sciences, Nagasaki University, 1-12-4 Sakamoto, Nagasaki-shi, Nagasaki 852-8523, Japan

E-mail: takatamu@nagasaki-u.ac.jp

Key words: STEAP4, focal adhesion kinase, CpG methylation, prostate cancer deficient mice shows that STEAP4 controls inflammatory response in adipocytes and loss of STEAP4 leads to a metabolic syndrome (3). STEAP4 is broadly expressed in various normal tissues including placenta, lung, heart, prostate and adipose $(1,4)$. However, in contrast to STEAP1 whose expression is widely detected in many cancers (e.g. prostate, lung, bladder, pancreas, ovary, colon) $(5,6)$, a previous report demonstrated that many human cancer cells do not express STEAP4, although LNCaP, human androgendependent prostate cancer cells express STEAP4 (1). This may suggest that STEAP4 expression is deleterious in cancer progression but it remained unclear. In addition, the mechanisms of down-regulation of STEAP4 expression in cancer cells have not been studied. Such research may elucidate a new approach to tumor malignancy and develop anti-tumor therapeutic strategy targeted to expression and function of STEAP4. Actually, we previously developed a dendritic cell-mediated immunization protocol and generated monoclonal antibodies against external portions of STEAP4 (7).

In this study, we investigated the possible roles of STEAP4 in cancer progression. Unexpectedly, we found that STEAP4 expression may inhibit anchorage-independent cell growth. This phenomenon may be mediated by the regulation of phospho-Y397 of focal adhesion kinase (FAK) by STEAP4. Furthermore, we found that $\mathrm{CpG}$ sequences in STEAP4 promoter region were frequently methylated in androgen-independent prostate cancer cells (DU145), but not in androgen-dependent prostate cancer cells (LNCaP). Demethylation treatment such as 5-aza-2-deoxycytidine or 5aza-cytidine induced STEAP4 expression in DU145, suggesting the possibility that STEAP4 expression in more aggressive in prostate cancer cells is in part epigenetically regulated by CpG methylation-mediated mechanism. Our results indicate a novel function of STEAP4 and provide evidence that STEAP4 may be involved in tumor malignancy.

\section{Materials and methods}

Cells. 293T cells were maintained in Dulbecco's modified Eagle's medium (DMEM) supplemented with $10 \%$ heatinactivated fetal calf serum (FCS). For construction of 293T cells stably expressing STEAP4, we used Flp-In system 
(Invitrogen, Calsbad, CA). Briefly, 293T cells were transfected with $\mathrm{pFRT} / \mathrm{lacZeo}$ and selected by Zeocin resistance. Next, these cells were transfected with pcDNA5/FRT-STEAP4 and pOG44, then selected by hygromycin resistance. The colonies of $293 \mathrm{~T}$ cells stably expressing STEAP4 were picked up from culture dishes. LNCaP and DU145 were purchased from RIKEN BioResource Center (Tukuba, Japan) and cultured in RPMI-1640 supplemented with 10\% heatinactivated FCS.

Immunofluorescence staining. The staining procedure was described previously (7). As primary antibody for antiSTEAP4 staining, 4F2 mAb was used. The FITC-conjugated anti-mouse IgG (Jackson Immunoresearch, West Grove, PA) was used as secondary antibody.

Construction of the expression plasmid for STEAP4-GFP fusion protein. STEAP4-GFP fusion construct was generated by in frame ligation of C-terminal of STEAP4 and Nterminal of GFP for which cDNAs were produced by PCR. The linker sequence between STEAP4 and GFP has a KpnI site. The primer sequences for STEAP4 with KpnI linker were 5'-GACCCACCATGGAGAAAACTTGTATAGA-3' and 5'-TCGGTACCGTGTTTTGAGTTCCTTTC-3'. The primer sequences for GFP with KpnI linker were 5'-GAG GTACCATGGTGAGCAAGGGCGAGG-3' and 5'-GACTT ACTTGTACAGCTCGTC-3'. The sequences of PCR products were confirmed by standard sequencing method using $\mathrm{ABI}$ PRISM 3100-Avant DNA sequencer (Applied Biosystems, Foster City, CA). The DNA fragment encoding STEAP4GFP fusion protein was inserted into pCAGGS, resulting in pCAGGS-STEAP4-GFP. The STEAP4-GFP protein in transfected 293T cells showed the same subcellular localization as the intact STEAP4 (data not shown).

Anchorage-independent cell culture. For soft agar colony formation assays, cells $\left(4 \times 10^{2}\right)$ were suspended in $0.33 \%$ agar in DMEM 10\% FCS, poured onto plates containing a layer of $0.5 \%$ agar in DMEM $10 \%$ FCS, and then cultured for 14 days. In some cases, $60 \mu \mathrm{g} / \mathrm{ml}$ anti-STEAP4 mAb (4F2) was added in both of upper and lower agar layers. The resulting colonies were counted with a colony being defined as $>20$ cells. Samples were assayed in triplicates and the experiment was repeated twice.

For suspension culture on poly-2-hydroxyethylmethacrylate (poly-HEMA)-coated dishes, $1 \times 10^{6}$ cells were plated into $60 \mathrm{~mm}$ poly-HEMA- (Sigma, St. Louis, MO) coated dishes. The poly-HEMA-coated dishes were prepared by applying $2 \mathrm{ml}$ of poly-HEMA solution $(12 \mathrm{mg} / \mathrm{ml}$ in $95 \%$ ethanol) onto the dishes, drying in tissue culture hood, followed by wash with PBS.

Western blotting analysis. The proteins in cell lysates were separated by SDS-PAGE and transferred to Immobilon membranes (Millipore, Bedford, MA). The Block-Ace (Snow Brand Milk Products, Tokyo, Japan) solution was used for blocking the membranes, and membranes were then reacted with 1:250 diluted anti-FAK (phosphoY397; BectonDickinson, San Jose, CA) antibody for $1 \mathrm{~h}$ at room temperature. After washing, the membranes were incubated with

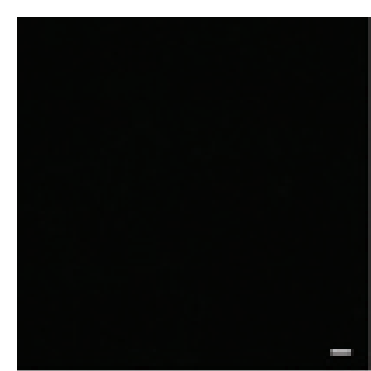

empty

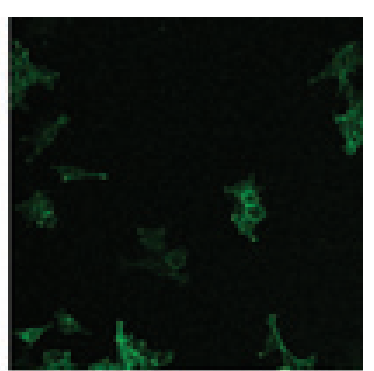

steap4
Figure 1. The expression of STEAP4 in 293T stable lines. The cells were stained with anti-STEAP4 mAb (4F2). Left: 293T-empty. Right: 293TSTEAP4. Scale bar $=20 \mu \mathrm{m}$.

horseradish peroxidase-conjugated goat anti-mouse IgG (Promega, Madison, WI). After washing, the membranes were immersed in ECL solution (GE healthcare, Piscataway, $\mathrm{NJ}$ ) and intensity of reactive band was analyzed using LAS1000 system (Fujifilm, Tokyo, Japan). Then, the bound antibodies were stripped from the membrane in 2\% SDS at $50^{\circ} \mathrm{C}$, and the membranes were incubated with 1:500 diluted anti-FAK (total; Becton-Dickinson) followed by the same procedure to detect phospho-Y397 FAK.

Immunoprecipitation. The cells were lysed in NP40 lysis buffer which consisted of $10 \mathrm{mM}$ Tris- $\mathrm{HCl}$ (pH 7.4), $150 \mathrm{mM}$ $\mathrm{NaCl}, 0.5 \mathrm{mM}$ EDTA and 1\% NP40 with PhosSTOP (Roche, Indianapolis, IN) and protease inhibitor cocktail set (Calbiochem, La Jolla, CA) for $15 \mathrm{~min}$ on ice. The cell extracts were prepared by centrifugation to remove cell debris. The cell extracts were mixed with anti-STEAP4 mAb (4F2) or anti-GFP antibody (Clontech, Palo Alto, CA) for $1 \mathrm{~h}$ at $4^{\circ} \mathrm{C}$ and protein G-Sepharose beads (GE Healthcare) were added and incubated for another $1 \mathrm{~h}$ at $4^{\circ} \mathrm{C}$. After washing, beads were suspended in SDS-PAGE sample buffer and boiled to release the bound proteins, which were subject to Western blot analysis.

Bisulfite genomic sequencing. The genomic DNA from human prostate cancer cells was isolated using a kit (MagExtractor, Toyobo, Osaka, Japan). The sodium bisulfite treatment of genomic DNA was done according to the manufacturer's instruction (BisulFast DNA modification kit for methylated DNA detection, Toyobo). The converted DNA was used as template for PCR. The condition of PCR was $94^{\circ} \mathrm{C} 25 \mathrm{sec}$, $52^{\circ} \mathrm{C} 25 \mathrm{sec}, 72^{\circ} \mathrm{C} 25 \mathrm{sec}$ of 40 cyles using Ex Taq hot start version (Takara, Shiga, Japan). The primer sequences for region-1 was 5'-GTTTTTTTAGGAAAGGGGGATTTT-3' and 5'-TAAAAATCCCAACAACTAAAAAAA-3'. The primer sequences for region-2 was 5'-ATTTTTTTAGGAA ATAAGTTTTTT-3' and 5'-AAAATAACCCACCTAA AAAAAAAA-3'. The PCR product was subcloned into pGEM-T easy vector (Promega) and the independent plasmid clones were sequenced using ABI PRISM 3100 sequencer.

$R T$-PCR. The cells were incubated with $10 \mu \mathrm{M}$ 5-aza-2deoxycytidine (5-aza-dC; Sigma). This dose of 5-aza-dC showed slowing of cell proliferation but not complete cell 


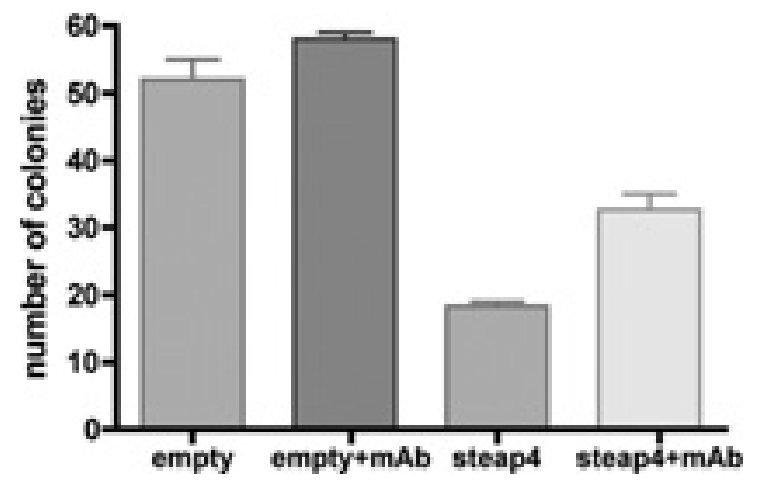

Figure 2. 293T-STEAP4 cells exhibited decreased colony formation in soft agar. 293T-empty or -STEAP4 cells were grown in soft agar conditions with or without anti-STEAP4 mAb (4F2). The average numbers of colonies \pm SEM are indicated. $\mathrm{p}<0.05$; empty vs. steap4, $\mathrm{p}<0.05$; steap4 vs. steap $4+$ $\mathrm{mAb}, \mathrm{p}>0.05$; empty vs. empty $+\mathrm{mAb} . \mathrm{p}<0.05$; significant, $\mathrm{p}>0.05$; not significant in t-test.

death (data not shown). Next day (day 1), the medium with fresh 5-aza-dC was replaced. On day 3, total RNA was isolated using Isogen (NipponGene, Toyama, Japan). In some cases, $1 \mu \mathrm{M}$ 5-aza-cytidine (5-aza-C; Sigma) was used. The cDNA was produced using oligo(dT) primer and Superscript II (Invitrogen). The PCR cycle was $94^{\circ} \mathrm{C} 30 \mathrm{sec}, 50^{\circ} \mathrm{C} 30 \mathrm{sec}$, $72^{\circ} \mathrm{C} 30 \mathrm{sec}$ ( 35 cycles for STEAP4, 25 cycles for GAPDH). The primer sequences for STEAP4 were 5'-ACACAGAACAC ACGCTCCTT-3' and 5'-TGACGGACTATGAGCCAGGA-3'. The primer sequences for GAPDH were 5'-GAGCTGAA CGGGAAGCTCAC-3' and 5'-TGTCATACCAGGAAATG AGC-3'.

\section{Results}

STEAP4 decreases soft agar colony formation. To explore possible function of STEAP4, 293T cells stably expressing STEAP4 (293T-STEAP4) were constructed. The immunostaining analysis showed that the expression of STEAP4 was mainly detected in cell surface of 293T-STEAP4 cells, although 293T-empty cells, in which empty vector was stably transfected, were not significantly stained (Fig. 1).

The proliferation of 293T-STEAP4 cells was the same as the 293T-empty cells under adherent culture condition (data not shown). However, in soft agar colony formation assay, the number of colonies in 293T-STEAP4 was significantly decreased compared with the 293T-empty (Fig. 2). The inhibition of colony formation in 293T-STEAP4 was reversed by anti-STEAP4 monoclonal antibody (Fig. 2). These results demonstrated that STEAP4 was able to inhibit anchorage-independent cell proliferation.

STEAP4 regulates $Y 397$ phosphorylation of FAK. The anchorage-independent cell growth is one of the most prominent features of cellular malignancy. The molecular mechanism of anchorage-independent cell growth is not fully understood, but previous studies suggest that FAK plays a critical role and phosphorylation of Y397 is important $(8,9)$. Therefore, we investigated the activation status of FAK in the presence of STEAP4. The culture of 293T cells in polyHEMA-coated dishes for $24 \mathrm{~h}$ resulted in $>90 \%$ trypan blue

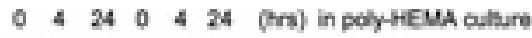

A
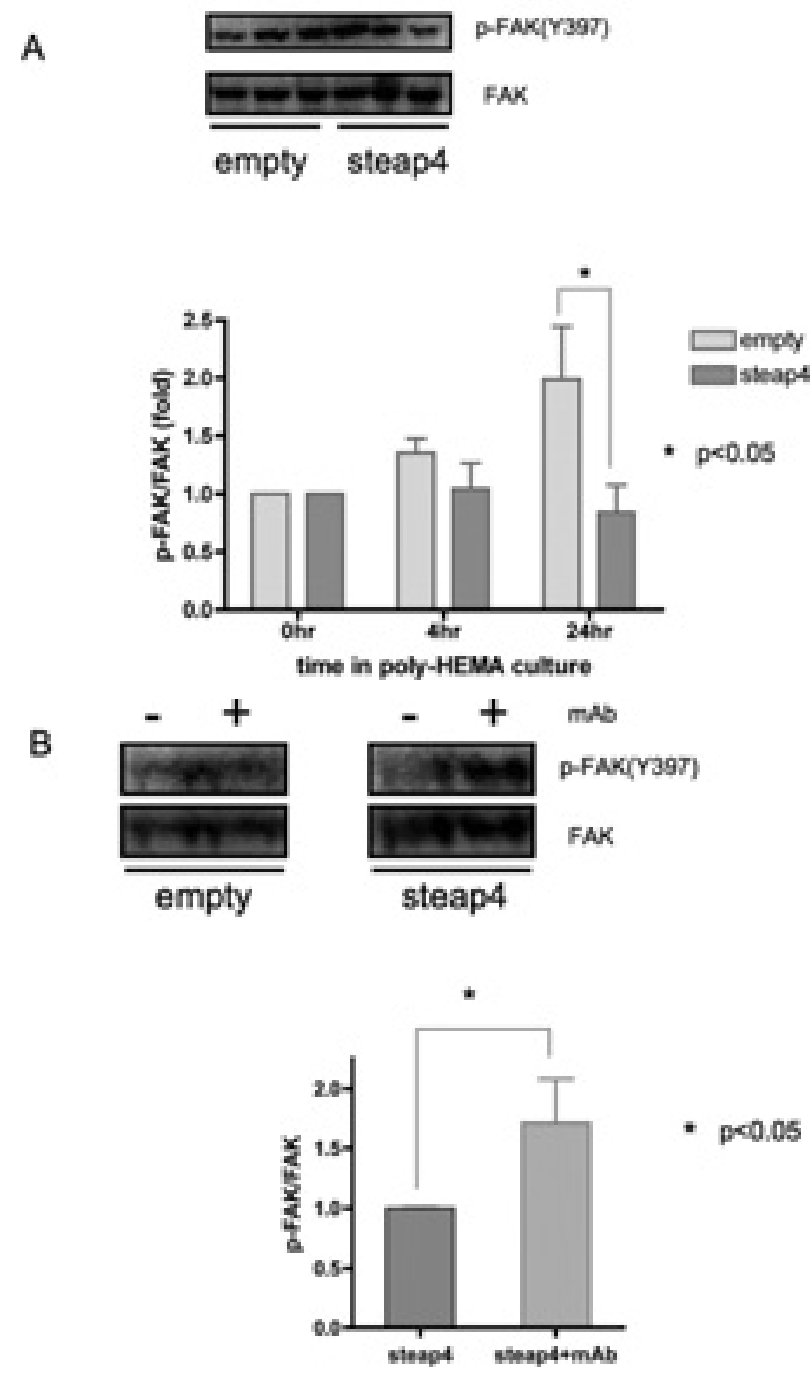

Figure 3. 293T-STEAP4 cells were unable to keep Y397 phosphorylation of FAK in poly-HEMA culture. (A) 293T-empty or -STEAP4 cells were nonadherently cultured in poly-HEMA-coated dishes for 4 and $24 \mathrm{~h}$, then collected and analyzed in Western blotting. The value of p-FAK/FAK was calculated as the band intensity of p-FAK/that of FAK. The value of p-FAK/ FAK at $0 \mathrm{~h}$ was set as 1.0 for fold activation. The average values of p-FAK/ FAK \pm SEM are indicated. The representative image of Western blotting is shown. (B) 293T-empty or -STEAP4 cells were non-adherently cultured in poly-HEMA-coated dishes for $24 \mathrm{~h}$ with or without $20 \mu \mathrm{g} / \mathrm{ml}$ anti-STEAP4 $\mathrm{mAb}$ (4F2), then collected and analyzed in Western blotting. The value of pFAK/FAK was calculated as the band intensity of p-FAK/ that of FAK. The value of $\mathrm{p}-\mathrm{FAK} / \mathrm{FAK}$ without antibody was set as 1.0 for fold activation. The average values of $\mathrm{p}-\mathrm{FAK} / \mathrm{FAK} \pm \mathrm{SEM}$ are indicated. The representative image of Western blot analysis is shown.

negative and $293 \mathrm{~T}$ cells are likely to be resistant to anoikis, although prolonged culture (e.g. 10 days) resulted in cell death. When 293T-empty cells were non-adherently cultured in poly-HEMA-coated dishes, Y397 phosphorylation of FAK was time-dependently increased (Fig. 3A). Such increase of Y397 phosphorylation in suspension culture condition of cancer cells was reported previously $(10,11)$. In contrast, Y397 phosphorylation of FAK in 293T-STEAP4 cells was not increased, but rather decreased in suspension culture (Fig. 3A). The anti-STEAP4 antibody enhanced Y397 phosphorylation in 293T-STEAP4 cells, although did not change in 293T-empty cells (Fig. 3B). Collectively, STEAP4 
A

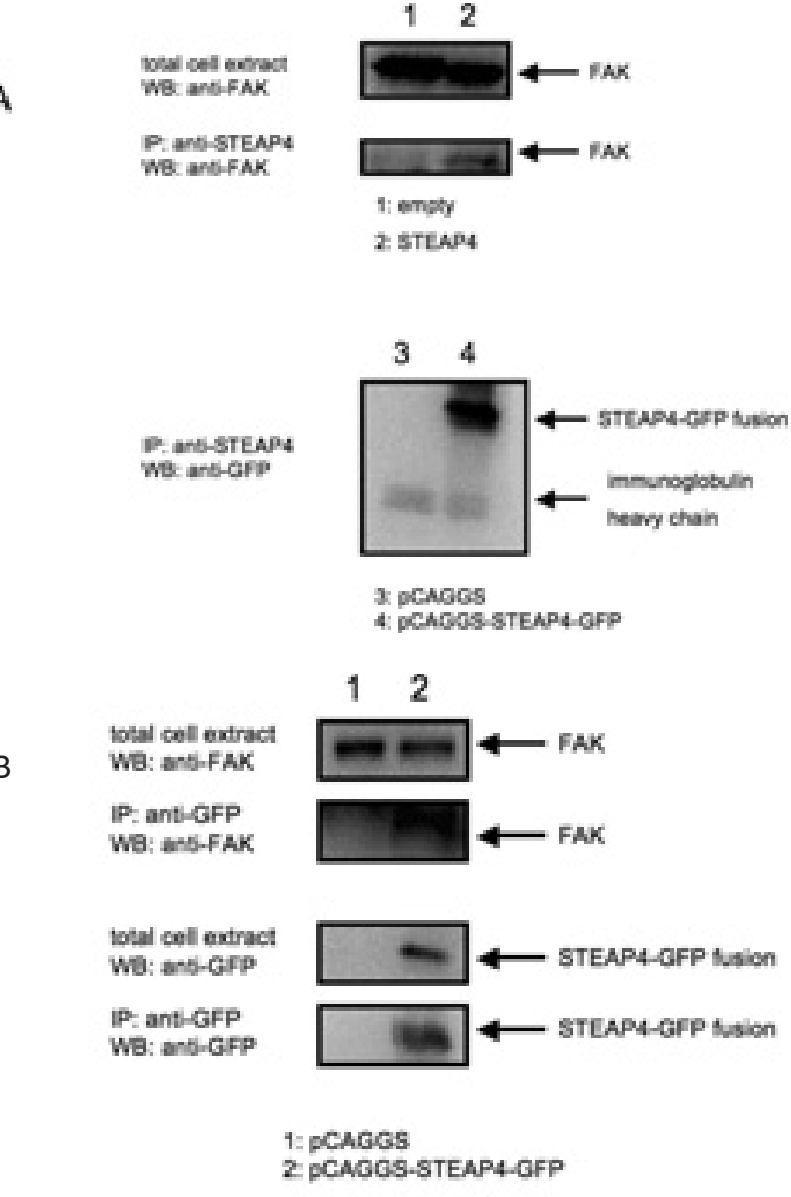

Figure 4. FAK associated with STEAP4. (A) The cell extracts of 293Tempty or -STEAP4 cells were immunoprecipitated using anti-STEAP4 antibody (4F2). The FAK protein was detected in Western blot analysis. Lane 1, 293-empty; lane 2, 293-STEAP4. As confirmation of immunoprecipitation using 4F2, the cell extracts of $293 \mathrm{~T}$ cells transfected with pCAGGS or pCAGGS-STEAP4-GFP were immunoprecipitated using 4F2. The STEAP4-GFP protein was detected in Western blotting. Lane 3, $293 \mathrm{~T}$ cells transfected with pCAGGS; lane 4, 293T cells transfected with pCAGGS-STEAP4-GFP. (B) The cell extracts of 293T cells transfected with pCAGGS or pCAGGS-STEAP4-GFP were immunoprecipitated using anti-GFP antibody. The FAK protein was detected in Western blotting. Lane1, 293T cells transfected with pCAGGS; lane 2, 293T cells transfected with pCAGGS-STEAP4-GFP.

could suppress Y397 phosphorylation of FAK in suspension culture condition, suggesting that this insufficient activation of FAK in suspension may explain inhibition of soft agar colony formation of SETAP4 expressing cells.

STEAP4 associates with FAK. The subcellular localization of STEAP4 protein is mainly plasma membrane and similar to FAK. To determine whether STEAP4 physically interacts with FAK, the extracts of 293T-STEAP4 cells were immunoprecipitated with anti-STEAP4 antibody and reacted with anti-FAK antibody for Western blot analysis. In Fig. 4A, FAK was shown co-immunoprecipitated with STEAP4, suggesting that STEAP4 interacts with FAK in 293TSTEAP4 cells. The molecular weight of STEAP4 (approximately $52 \mathrm{kDa}$ ) is almost same as IgG heavy chain and hard to discriminate in immunoprecipitation. Therefore, it was uncertain whether the anti-STEAP4 antibody was actually able to immunoprecipitate STEAP4 protein. Thus, we used an expression construct for STEAP4-GFP fusion protein. Because GFP is fused to C-terminal of STEAP4, STEAP4GFP is sized up in molecular weight and easy to discriminate with IgG heavy chain. Indeed, anti-STEAP4 mAb was used for immunoprecipitation analysis because STEAP4GFP fusion protein was clearly detected in immunoprecipitation using anti-STEAP4 mAb in 293T cells transfected with pCAGGS-STEAP4-GFP (Fig. 4A). Moreover, immunoprecipitation experiments using anti-GFP antibody demonstrated that STEAP4-GFP also associated with FAK (Fig. 4B). These results suggest that STEAP4 interacts with FAK and this association may be important to regulation of FAK function by STEAP4.

The expression of STEAP4 is down-regulated by $C p G$ methylation mechanism in human androgen-independent prostate cancer cells. In general, less aggressive prostate cancer cells grow androgen-dependently, but more aggressive prostate cancer cells turned androgen-independent. Previous studies suggested that STEAP4 expression is detected in LNCaP, androgen-dependent, but not detected in DU145, more aggressive and androgen-independent (1). Expression of many genes in cancer cells are regulated by epigenetic mechanism including $\mathrm{CpG}$ methylation $(12,13)$. Our results suggest that STEAP4 decreased anchorage-independent cell growth. Therefore, we decided to explore the possibility that STEAP4 expression might be down-regulated by $\mathrm{CpG}$ methylation in the course of more aggressive cancer.

As previously reported, STEAP4 mRNA expression was detected in human normal prostate and LNCaP, but not detected in DU145 (Fig. 5A). However, when DU145 cells were cultured in the presence of 5-aza-dC, demethylation reagent, STEAP4 expression in 5-aza-dC-treated DU145 cells was significantly increased in RT-PCR analysis (Fig. 5A). Furthermore, 5-aza-C, another demethylation reagent treatment gave a similar result in DU145 cells (Fig. 5A). In addition to mRNA expression level, immunofluorescence staining demonstrated increased STEAP4 expression in protein level by 5-aza-C treatment in DU145 (Fig. 5B). These results suggest that STEAP4 expression may be regulated by $\mathrm{CpG}$ methylation in DU145 cells.

CpG motifs within STEAP4 promoter region is frequently methylated in human androgen-independent prostate cancer cells. The analysis of genomic DNA sequence of STEAP4 gene locus (7q21.12) revealed that single-predicted $\mathrm{CpG}$ island exists around STEAP4 promoter region, encompassing 5 ' upstream sequence and part of first exon (Fig. 6A). This prompted us to investigate $\mathrm{CpG}$ methylation status in $\mathrm{LNCaP}$ and DU145 cells. The sequence analysis of bisulfite-treated genomic DNA demonstrated that almost no CpG methylation in STEAP4 promoter region was detected in $\mathrm{LNCaP}$ cells, but methylated $\mathrm{CpG}$ motifs were actually found in DU145 cells (Fig. 6B). The degree of methylation seemed to be higher in region-1, which is located in 5' upstream sequence, than region-2. These results suggest that $\mathrm{CpG}$ sequences in STEAP4 promoter region are frequently methylated, which may suppress STEAP4 expression in prostate cancer cells. 
A

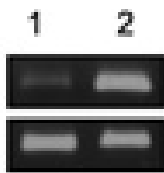

STEAP4

GAPDH

1: LNCaP

2: human normal prostate

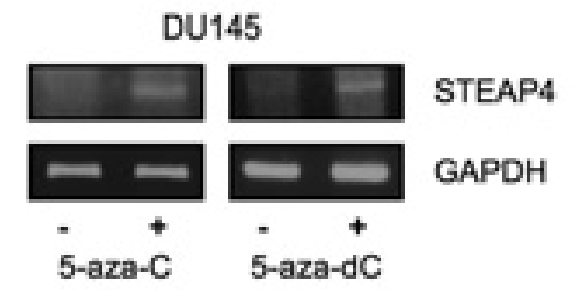

B
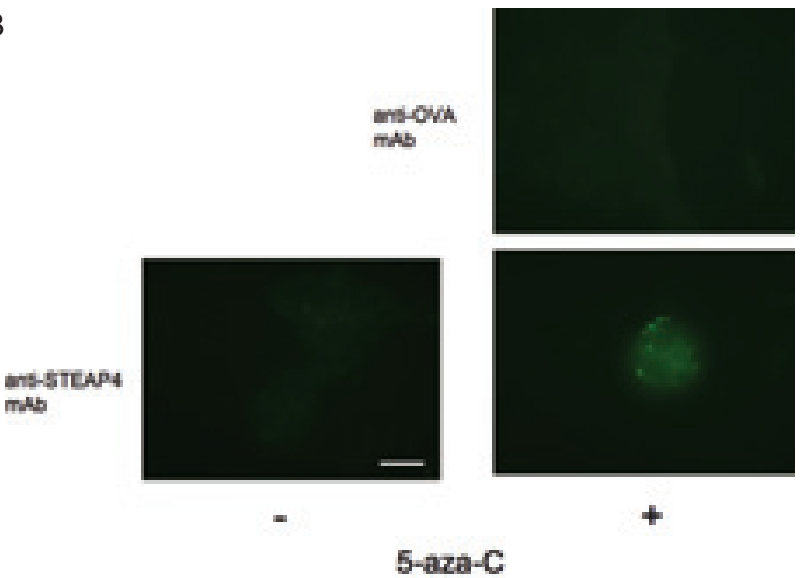

Figure 5. The demethylation treatment induced STEAP4 expression in DU145 cells. (A) RT-PCR analysis of STEAP4 expression in human prostate cancer cells. (B) Immunofluorescence staining of 5-aza-C-treated DU145 cells. For STEAP4 staining, anti-STEAP4 mAb (4F2) was used. As negative control, anti-OVA mAb was used. Scale bar $=20 \mu \mathrm{m}$.

\section{Discussion}

In this study, we investigated the possible roles of STEAP4 in cancer progression. Our results suggest that STEAP4 binds to FAK and regulates activation of FAK, which may affect the cell growth in soft agar. Moreover, our results demonstrate that methylation of $\mathrm{CpG}$ motifs in STEAP4 promoter region may down-regulate STEAP4 expression. Taken together, these data implicate a novel activity of STEAP4 in cancer progression.

The functions of FAK in cancer cells are diverse such as metastasis, invasion in addition to anchorage-independent proliferation. STEAP4 may modulate these properties of cancer cells through regulation of FAK activation. The many molecules relevant to FAK are known such as Src, PI-3K p85 subunit, Grb2, p130cas and paxillin. The mechanisms how STEAP4 regulates downstream signaling from Y397 phosphorylation of FAK remains to be elucidated. A previous report demonstrated that clonogenic proliferation was enhanced when STEAP4 gene was transduced, although the exact mechanism of STEAP4 activity remained unclear (1). This observation is inconsistent with our finding, but the difference of experimental condition (e.g. adherent or anchorage-independent) should be considered. We found Y397 phosphorylation of FAK in 293T-STEAP4 tends to be higher than in 293T-empty under adherent culture conditions (data not shown), which may be advantageous in clonogenic prolifearion under anchorage-dependent culture condition. Several binding proteins to STEAP3 (also called TSAP6), belonging to STEAP family, like STEAP4, were identified using yeast two-hybrid system, suggesting that STEAP3 played a role in regulation of cell proliferation (14). In this study, we showed STEAP4 binds to FAK. Moreover, the prediction software analysis (NetPhosK; http://www.cbs. dtu.dk/services/NetPhosK/) demonstrates that human

A
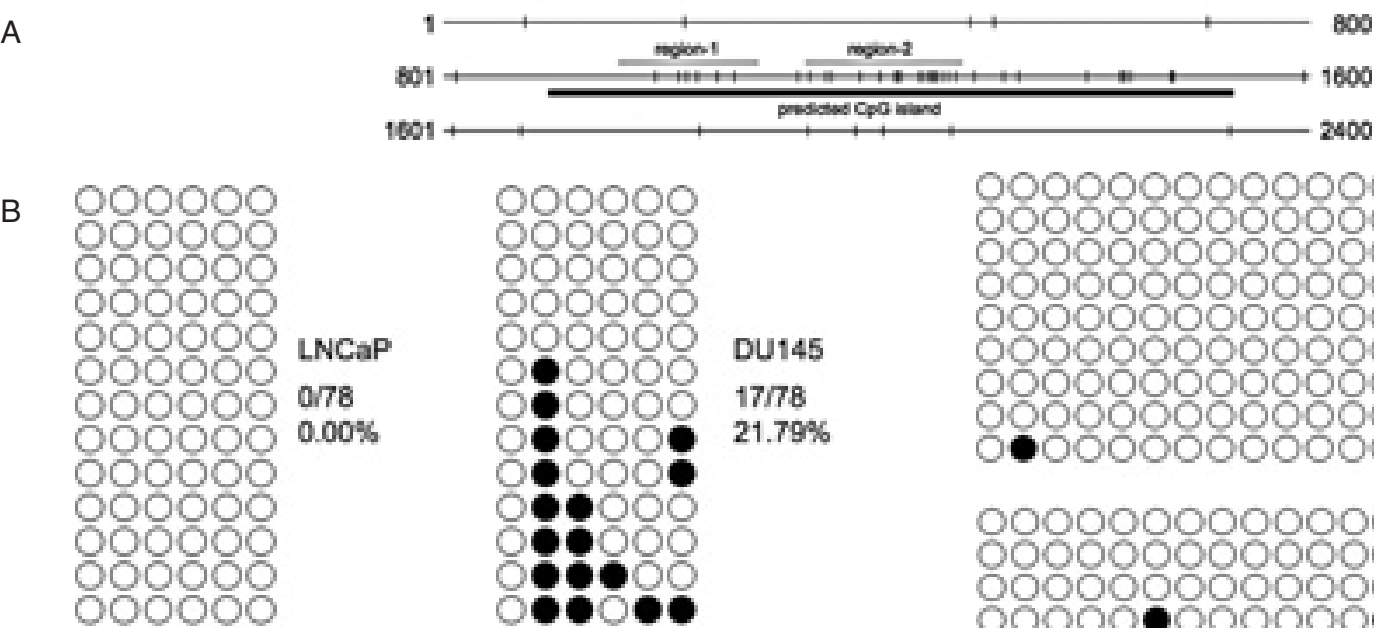

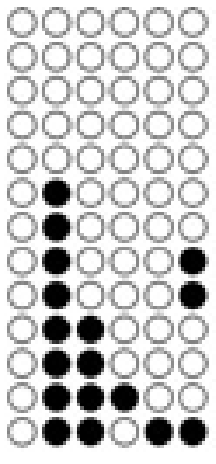

region-1
DU145

$17 / 78$

$21.79 \%$

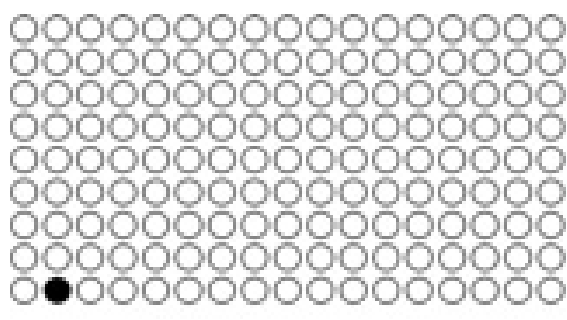

LNCaP

$1 / 153$

$0.65 \%$

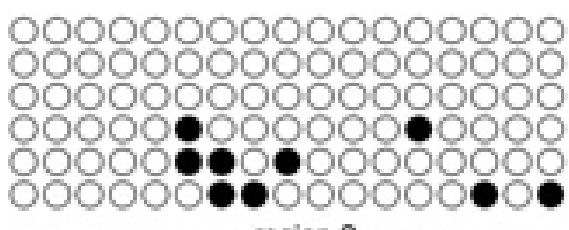

DU145

$9 / 102$

$8.82 \%$

Figure 6. CpG methylation in STEAP4 promoter region in DU145 cells. (A) The schematic depiction of CpG island in STEAP4 promoter region. The predicted $\mathrm{CpG}$ island and regions analyzed for bisulfite genomic sequencing are indicated. For prediction of $\mathrm{CpG}$ island, $\mathrm{CpG}$ Island Searcher (http://cpgislands.usc.edu/) was used. The position of predicted transcription start site is 1200. The vertical bars are CpG motifs. (B) The methylation status of CpG motifs in region-1 and region-2 in LNCaP and DU145 cells. The percentage of methylated CpG was calculated. Open circle: unmethylated CpG. Closed circle: methylated $\mathrm{CpG}$. 
STEAP4 has several putative phosphorylation sites including Src, p38 MAP kinase, GSK3 etc. This suggests that STEAP4 may be a target for post-transcriptional modification such as phosphorylation. A more detailed molecular interplay between STEAP4 and other proteins needs to be understood for STEAP4 function in cancer cells.

Our study demonstrated that $\mathrm{CpG}$ methylation was actually detected in STEAP4 promoter region and demethylation treatment induced STEAP4 expression in DU145 cells. However, the $\mathrm{CpG}$ methylation status analysis of the clinical tumor samples with various stages including prostate cancer is necessary for the comprehensive understanding of the significance of $\mathrm{CpG}$ methylation in STEAP4 promoter region in cancer progression. $\mathrm{CpG}$ methylation as a sole mechanism in down-regulation of STEAP4 in prostate cancer cells is unlikely. In this study, CpG methylation was analyzed, but other epigenetic mechanism such as histone modification also should be considered. It may be probable that expression of transcription factors that could be involved in STEAP4 expression are epigenetically regulated although the STEAP4 promoter region is not epigenetically modified. The detailed regulation mechanism of STEAP4 expression in cancer cells has not been revealed yet, and various possibilities including epigenetic mechanism, which is demonstrated in this study, should be considered.

\section{References}

1. Korkmaz CG, Korkmaz KS, Kurys P, et al: Molecular cloning and characterization of STAMP2, an androgen-regulated six transmembrane protein that is overexpressed in prostate cancer. Oncogene 24: 4934-4945, 2005.

2. Ohgami RS, Campagna DR, McDonald A and Fleming MD: The Steap proteins are metalloreductases. Blood 108: 1388-1394, 2006.
3. Wellen KE, Fucho R, Gregor MF, et al: Coordinated regulation of nutrient and inflammatory responses by STAMP2 is essential for metabolic homeostasis. Cell 129: 537-548, 2007.

4. Arner P, Stenson BM, Dungner E, Naslund E, Hoffstedt J, Ryden $\mathrm{M}$ and Dahlman I: Expression of six transmembrane protein of prostate 2 in human adipose tissue associates with adiposity and insulin resistance. J Clin Endocrinol Metab 93: 2249-2254, 2008.

5. Hubert RS, Vivanco I, Chen E, et al: STEAP: a prostate-specific cell-surface antigen highly expressed in human prostate tumors. Proc Natl Acad Sci USA 96: 14523-14528, 1999.

6. Challita-Eid PM, Morrison K, Etessami S, et al: Monoclonal antibodies to six-transmembrane epithelial antigen of the prostate- 1 inhibit intercellular communication in vitro and growth of human tumor xenografts in vivo. Cancer Res 67: 5798-5805, 2007.

7. Tamura T and Chiba J: Production of antibodies against multipass membrane proteins expressed in human tumor cells using dendritic cell immunization. J Biomed Biotechnol 2009: 673098, 2009

8. Parsons JT: Focal adhesion kinase: the first ten years. J Cell Sci 116: 1409-1416, 2003.

9. van Nimwegen MJ and van de Water B: Focal adhesion kinase: a potential target in cancer therapy. Biochem Pharmacol 73: 597-609, 2007.

10. Johnson TR, Khandrika L, Kumar B, et al: Focal adhesion kinase controls aggressive phenotype of androgen-independent prostate cancer. Mol Cancer Res 6: 1639-1648, 2008.

11. Liu G, Meng X, Jin Y, et al: Inhibitory role of focal adhesion kinase on anoikis in the lung cancer cell A549. Cell Biol Int 32: 663-670, 2008.

12. Baylin SB and Ohm JE: Epigenetic gene silencing in cancer - a mechanism for early oncogenic pathway addiction? Nat Rev Cancer 6: 107-116, 2006.

13. Jones PA and Baylin SB: The epigenomics of cancer. Cell 128: 683-692, 2007.

14. Passer BJ, Nancy-Portebois V, Amzallag N, et al: The p53inducible TSAP6 gene product regulates apoptosis and the cell cycle and interacts with Nix and the Myt1 kinase. Proc Natl Acad Sci USA 100: 2284-2289, 2003. 